\title{
A Prospective Outcomes Study of Patients Undergoing Intradiscal Electrothermy (IDET) for Chronic Low Back Pain
}

\author{
Peter C. Gerszten*, MD, MPH, William C. Welch ${ }^{* *}$, MD, FACS, Paula M. McGrath", RN, BSN \\ and Shari L. Willis\#, RN, BSN
}

This prospective, non-randomized clinical trial was designed to determine the clinical outcome of patients who underwent Intradiscal Electrotherapy (IDET) for the treatment of chronic discogenic low back pain.

Twenty-seven consecutive patients undergoing IDET were prospectively evaluated. All patients, as determined by provocative discography and/or MRI, had discogenic disease with chronic low back pain and were nonresponsive to conservative treatment for at least 6 months. The mean pre-operative duration of symptoms was 38 months. The American Association of Neurological Surgeons/Congress of Neurological Surgeons Joint Section Lumbar Disc Herniation Study Questionnaire, which includes the Oswestry Low Back Pain Disability Questionnaire and the Short Form (SF) -36 Health Status Questionnaire, was used. The follow-up endpoint for all patients was one year.

Seventy-five percent of patients improved based upon the
Oswestry Low Back Pain Disability Questionnaire while only $48 \%$ of patients were found to improve on the SF-36 Survey. The SF-36 Bodily Pain Subscale did improve relative to other subscales in $52 \%$ of patients. There was no relationship found between outcome and duration of symptoms $(p=.32)$, number of levels treated $(p=.20)$, or worker's compensation $(\mathrm{p}=.38)$. There were no complications that resulted from the IDET treatment.

IDET was found to be effective in $75 \%$ of patients in improving their chronic low back pain. This did not translate into a significant improvement in the SF-36 survey scores. The risks are negligible, and recovery time is minimal. The procedure may be useful in selected patients who would otherwise undergo an interbody fusion procedure.

Keywords: Discogenic pain, intradiscal electrothermy, low back pain

Over the years, a number of intradiscal techniques to either shrink or remove disc material believed to be causing lumbar pain and/or radiculopathy have been described (49, 13-18). These techniques include interbody fusion, posterior-lateral fusion, microdiscectomy, arthroscopic discectomy, automated percutaneous lumbar discectomy, chymopapain, as well as other procedures (11-21).

Intradiscal Electrothermy (IDET) is a technique that involves the percutaneous insertion of a thermal resistance probe with controlled heating of the disc material $(7,12)$. Several clinical studies of IDET showed improved clinical outcomes (4-8). The purpose of this study was to report a prospective longitudinal cohort study from a single institution's experience using IDET for the treatment of discogenic low back pain.

\section{METHODS}

Twenty-seven patients with chronic low back pain (back pain for longer than six months) were consecutively 
enrolled in a non-randomized, longitudinal, observational trial over the study period (January 2000 - October 2001). This study was not corporate sponsored. Nine patients reported only low back pain that was not lateralized and had no leg symptoms (neither radicular nor non-radicular). The primary objective was to assess the patients' clinical outcomes and the treatment of their discogenic lower back pain. This study was funded from internal sources independent of any relationship to the manufacturer of the IDET catheter.

\section{Inclusion Criteria}

- Unremitting, persistent low back pain of at least six months continuous duration

- Low back pain greater than leg pain

- Discogenic back pain was defined as pain with tasks requiring axial loading of the spine and relief of pain with recumbency

- Evidence of discogenic disease on magnetic resonance imaging (MRI) scan including disc desiccation, high-intensity zones in the disc, disc rupture, loss of disc height, or concordant provocative discography

- Failure of non-surgical therapies in the prior six months including physical therapy, non-steroidal antiinflammatory medicines, and epidural steroidal treatments

\section{Exclusion Criteria}

- Evidence of instability on imaging studies (greater than $5 \mathrm{~mm}$ of subluxation)

- Active infection

- Malignancy

- Metabolic disorder that would preclude appropriate follow-up and participation

\section{Patient Management}

Patients presenting with lower back pain with or without leg symptoms were evaluated in a neurosurgery spine specialty clinic by a fellowship trained neurosurgical spine surgeon. A medical history was ascertained from each patient and followed by a complete physical and neurological exam. The surgeons then reviewed and discussed all treatment options with the patients and his/ her family. If a patient was determined to have lower back pain of discogenic origin and met inclusion and exclusion criteria of the trial, then he/she was offered the IDET procedure. Nineteen patients underwent provo- cative discography. Eight patients had only MR imaging. Those patients had refused the provocative discogram and had significant MR findings at only a single level.

Several patients were offered the IDET procedure because they were felt to be at too high a medical risk for a fusion procedure. Other patients could not afford to take time off from work to recover from a fusion procedure. Each of the 27 patients determined eligible to participate in the trial signed an informed consent approved by the Institutional Scientific Review Board of the University of Pittsburgh. No study related procedures had taken place prior to obtaining the patients' informed consent.

\section{IDET Technique}

A standardized IDET technique was performed on all patients. All procedures were performed in prone position in the operating room. Intravenous sedation was given and C-arm fluoroscopy was utilized to obtain anteriorposterior and lateral images. The treatment level was localized and local anesthesia was applied to the skin $6-$ $9 \mathrm{~cm}$ lateral to the midline. The 17 -gauge needle and stylet were directed toward the center of the disc under fluoroscopic guidance, and the annulus was punctured. The thermal-resistance catheter was inserted through the needle into the disc. The catheter was coiled within the disc as it was deflected by the annular fibers. The tip of the catheter was directed to the posterior aspect of the disc in such a manner that the heating elements of the catheter remained on the symptomatic side. Therefore, the needle was inserted on the side contralateral to the patient's pain. The catheter temperature was increased along an electronically programmed protocol over 13 minutes to $90^{\circ} \mathrm{C}$ and allowed to remain at that temperature for four minutes. Antibiotics were not injected into the disc space. The catheter and needle were then removed as a single unit.

Patients with multi-level disease underwent treatment at other involved level(s) using the same protocol. New catheters and needles were used at each level. Patients with only back pain and no leg pain underwent treatment on the opposite disc side (i.e. bilateral) as well. The patients were discharged after two hours of observation. Following the procedure, patients were instructed to resume their usual activities as tolerated after 24 hours.

\section{Outcomes Measures and Data Collection}

Quality of life and disability data collections were taken 
at a baseline prior to the procedure. The outcome instrument used was the American Association of Neurological Surgeons/Congress of Neurological Surgeons Joint Section Lumbar Disc Herniation Study Questionnaire that included the Short Form (SF)-36 Health Status Questionnaire as well as the Oswestry Low Back Pain Disability Questionnaire. The instruments were obtained at baseline, six weeks, three months, and one year after treatment. A visual analog pain score was not used for this study.

An online data collection system (Outcomes Sciences, Boston, MA) was utilized throughout this study for data storage and analysis. Once the study subjects completed the required questionnaires, they were returned to the study site, and the data were entered by the study coordinator.

\section{Statistical Analysis}

The SF-36 and the Oswestry Low Back Pain Disability Questionnaire summary scores were tabulated. Pre- and post-treatment mean scores were ascertained. The one year follow-up scores were used for all patients for final statistical analysis.

\section{RESULTS}

\section{Demographics}

The study group consisted of 27 patients (15 men, 12 women; mean age 41 years). The mean duration of symptoms was 38 months (range 6 months to 15 years). There were 8 patients with private insurance and 19 patients receiving workers' compensation. Sixteen (16) patients were treated at one disc level and 11 patients at two or more disc levels.

A total of 38 catheters were inserted. Six (6) catheters were inserted at the L3-4 disc space, 17 catheters at the L4-5 disc space and 15 catheters at the L5-S1 disc space. In 18 patients the catheter was inserted from only one side and in 9 patients the catheter was inserted from both sides.

\section{Outcomes}

The SF-36 was separated into the Physical Functioning Subscale, Bodily Pain Subscale, and the Role Functioning (Physical) Subscale (22). In each subscale, the lower the score, the more severe are the patient's symptoms. A significant improvement was defined as an improvement $>=7$ points based upon the methodology of previous authors $(4,5,7)$. At one year, forty-five percent $(47 \%)$ of patients reported a significant improvement on the SF-36 survey (Table 1). There was a $41 \%$ improvement on the SF-36 Bodily Pain Subscale in relation to the other subscales for all patients. An improvement was indicated if the scores increased by at least 7 points.

For the Oswestry Low Back Pain Disability Questionnaire, a lower score indicates less severe symptoms (23). Seventy-five percent $(75 \%)$ of patients had improvement of their symptoms following the IDET procedure based upon this questionnaire. There was no relationship found between outcome and duration of symptoms $(P=.32)$, the number of levels treated $(P=.20)$, or receipt of workers' compensation $(P=.38)$.

\section{Complications}

No serious complications occurred. There were no nerve root injuries or infections that occurred. Cerebral spinal fluid was visualized in one case. That patient was discharged to home without consequence. In one case, the catheter was unable to be threaded into the disc space secondary to scar tissue from a previous discectomy. This procedure was aborted and the patient subsequently

Table 1. Results: Outcomes pre-and post-treatment comparisons

\begin{tabular}{|c|c|c|c|}
\hline Scales & Baseline mean score & Post-treatment mean score & $\%$ change \\
\hline SF-36 physical functioning subscale & 32 & 47 & $47 \%$ \\
\hline SF-36 bodily pain subscale & 27 & 38 & $41 \%$ \\
\hline SF-36 role functioning-physical & 5 & 16 & $220 \%$ \\
\hline Oswestry low back pain questionnaire & 34 & 30 & $12 \%$ \\
\hline Neurogenic symptoms & 15 & 14 & $7 \%$ \\
\hline
\end{tabular}


underwent an anterior lumbar interbody fusion with significant improvement in his low back pain. This patient was not considered an IDET success.

\section{DISCUSSION}

The current study was designed to determine the outcome in a group of patients who underwent the IDET procedure for chronic low back pain who met the conventional clinical criteria for interbody fusion surgery $(9,20,24$, 25). For a variety of reasons, these patients were treated with an intradiscal thermal catheter. This was not a randomized study. The study cohort chose the IDET procedure as an alternative to spinal fusion. The study group was evaluated before treatment and at 12 months follow-up using both the SF-36 and the Oswestry Low Back Pain Disability Questionnaire.

The study patients represented a chronic group, with an average symptom duration of over three years. This study found no relationship between the duration of symptoms and outcome of the treatment. Based upon the Oswestry Low Back Pain Disability Questionnaire, $75 \%$ of patients demonstrated an improvement in their low back pain complaints. Cointervention was kept to a minimum in this patient group and consisted of nonoperative treatments to which they already had been exposed without success before IDET treatment.

During the duration of the study, all patients in the authors' clinical practice who underwent the IDET procedure were entered into the study. No patient was treated outside of the study. Therefore, selection bias was controlled for. Only $52 \%$ of patients demonstrated improvement based upon the SF-36 Bodily Pain Subscale. For this study, an improvement of 7 points or more on the SF-36 was used to determine a successful outcome. The authors chose this improvement criteria in order to compare our results to those of other reported series $(4,5,7)$. It is unclear as to the validity of the SF-36 subscales in determining improvements in intervention for low back pain.

In contrast, $75 \%$ of patients demonstrated an improvement in the Oswestry Low Back Pain Disability Questionnaire at one year follow-up. It is the authors' contention that the Oswestry outcomes instrument is more sensitive and valid a measure of low back pain improvement than the SF-36 instrument. It is unlikely that this chronic low back pain population would achieve spontaneous improvement in their symptoms to this extent during the follow-up period. There was no statistical relationship found between the duration of symptoms and the success of the IDET procedure in alleviating low back pain.

Although this series is relatively small, no statistically significant relationship was found between outcome and whether or not the patients were ensured through the workers' compensation system. These patients fared no differently than patients with private payers. This finding is similar to the results of another IDET series $(4,5)$.

There are several limitations to the study. The ideal clinical evaluation of the IDET procedure would consist of a blinded study in which half of the study cohort would be randomized to one of two groups: treatment or sham procedure. Another study might involve the randomization to either the IDET or interbody fusion procedure. The first study would be almost impossible to undertake at our institution. First, our patient population would never consent to enter such a study. Second, our Institutional Scientific Review Board would never approve such a study. Therefore, we are left with a prospective cohort observational study.

For this study, we intentionally did not report the patients' one-year employment status as an individual variable. We feel that employment status as an outcomes measure is extremely flawed for this particular patient cohort. Over two-thirds of the patients were involved in work-related injuries. Return to work status in workers' compensation cases is often complex and not directly related to the clinical status of the individual patient. Return to work status often is determined by the employer's ability to accommodate the patient. For many of our heavy labor employed patients, patients are not allowed to return to work until they are "100\%". It becomes difficult to determine what work the patient is actually doing compared to their pre-morbid condition. In addition, legal suits against employers for benefits and lost wages complicates return to work status.

The authors' have now performed over 75 intradiscal electrothermal annuloplasty procedures. The complication rate is essentially zero with no adverse events or worsening of the baseline clinical condition at one year follow-up. Recovery time is minimal, especially compared to an interbody fusion procedure.

It is unlikely that the study patients' outcome improvement could be attributed to differences in postoperative management that they received. There was no relationship found between the duration of symptoms and a clinically 
significant improvement. The study patients were not exposed to new treatments that they had not been exposed to prior to the IDET procedure.

\section{CONCLUSION}

This study represents a prospective outcomes evaluation of a group of patients with low back pain who met the conventional criteria for interbody fusion surgery, but who instead underwent the IDET treatment. IDET was found to be effective in $75 \%$ of patients by improving their low back pain based upon the Oswestry Low Back Pain Disability Questionnaire. Only one half of patients had a significant improvement based upon the Medical Outcomes Health Survey (SF-36). Outcome was not dependent upon number of levels treated, duration of symptoms, or workers' compensation. The risks are negligible, and recovery time is minimal. The procedure may be useful in patients who would otherwise undergo an interbody fusion procedure.

\section{REFERENCES}

1. Coppes M, Marani E, Thomeer R et al. Innervation of 'painful' lumbar discs. Spine 1997; 22:2342-2350.

2. Schwarzer A, April C, Derby R et al. The relative contributions of the disc and zygapophyseal joint in chronic low back pain. Spine 1994; 19:801-806.

3. Manchikanti L, Singh V, Pampati VS et al. Evaluation of the relative contributions of various structures in chronic low back pain. Pain Physician 2001; 4:308316.

4. Saal JS, Saal JA. Intradiscal electrothermal treatment (IDET) for chronic discogenic low back pain: A prospective outcome study with minimum one year follow-up. Spine 2000; 25:2262-2267.

5. Saal JA, Saal JS. Intradiscal electrothermal therapy for the treatment of chronic discogenic low back pain. Clin Sports Med 2002; 21:167-187.

6. Derby $\mathrm{R}$, Eek $\mathrm{B}$, Chen $\mathrm{Y}$ et al. Intradiscal Electrothermal Annuloplasty (IDET): A novel approach for treating chronic discogenic back pain. Neuromodulation 2000; 3:82-88.

7. Karasek M, Bogduk N. Twelve month follow-up of a controlled trial on intradiscal thermal annuloplasty for back pain due to internal disc disrupture. Spine 2000; 25:2601-2607.

8. Singh V. Intradiscal electrothermal therapy: A preliminary report. Pain Physician 2000; 3:367-373.

9. Vamvanij V, Fredrickson B, Thorpe JM et al. Surgical treatment of internal disc disruption: An outcome study of four fusion techniques. J Spinal Disord 1998;
11:375-382.

10. Cherkin DC, Deyo RA, Street JH et al. Predicting poor outcomes for back pain seen in primary care using patients' own criteria. Spine 1996; 21:29002907.

11. Onik G, Maroon J, Davis GW. Automated percutaneous discectomy at the L5-S1 level. Use of a curved cannula. Clinical Orthop 1989; 238:71-76.

12. Welch WC, Gerszten PC, McGrath P. Intradiscal electrothermy: Indications, techniques, and clinical results. Clin Neurosurg 2001; 48:219-225.

13. Sharps L, Isaac Z. Percutaneous disc decompression using Nucleoplasty®. Pain Physician 2002; 5:121126.

14. Singh V, Piryani C, Liao K et al. Percutaneous disc decompression in the treatment of chronic discogenic pain. Pain Physician 2002; 5:250-259.

15. Choy D. Percutaneous laser disc decompression (PLDD): Twelve years' experience with 752 procedures in 518 patients. J Clin Laser Med Surg 1998; 16:325-331.

16. Mochida J, Tos E, Nomura T, Nishimura K. Department of Orthopaedic Surg, Tokai University, Japan. The risks and benefits of percutaneous nucleotomy for lumbar disc herniation: a 10 -year longitudinal study. J Bone Joint Surg (Br) 2001; 83:501-505.

17. Gogan WJ, Fraser RD. Chymopapain. A ten-year, double-blind study. Spine 1992; 1:388-394.

18. Case RB, Choy DS, Altman P. Change of intradisc pressure versus volume change. J Clin Laser Med Surg 1995; 13:143-147.

19. Fritzell P, Hagg O, Wessberg P et al. Lumbar fusion versus nonsurgical treatment for chronic low back pain: A multicenter randomized controlled trial from the Swedish lumbar spine study group. Spine 2001; 26:2521-2532

20. Turner JA, Ersek M, Herron L et al. Patient outcomes after lumbar spinal fusions. JAMA 1992; 268:907911.

21. Kambin P. Alternative to open lumbar discectomy: Arthroscopic microdiscectomy. In Lange A (ed). Operative Spinal Surgery, Stanford, 1999.

22. Ware JE. SF-36 health survey update. Spine 2001; 25:3130-3139.

23. Fairbank JC, Pynsent PB. The Oswestry Disability Index. Spine 2000; 25:2940-2952.

24. Ray CD. Threaded fusion cages for lumbar interbody fusions: An economic comparison with $360^{\circ}$ fusions. Spine 1997; 22:681-685.

25. Zdeblick TA. A prospective, randomized study of lumbar fusions: Preliminary results. Spine 1993; 18:983-991 